\title{
A Systematic Review Study on the Communication between Diabetes and Cancer: The Serious Risk of Cancer in Diabetic Patients
}

\section{Mostafa Madmoli ${ }^{1 *}$}

Emergency Medical Technician, Dezful University of Medical Sciences, Dezful, Iran.

\begin{abstract}
Introduction: Diabetes is a chronic metabolic disease which accounts for 15 percent of healthcare spending in the United States. Patients with diabetes have been reported to increase the risk of cancer by 20 to 25 percent compared to those without diabetes. In this study, we intend to examine the relationship between these two diseases by examining several studies. Therefore, this systematic review was conducted to determine the relationship between diabetes and cancer and the risk of developing cancer in diabetic patients.

Materials and Methods: This study is a systematic review in order to achieve the goal of the study and to enhance the accuracy of its study and comprehension, this integrated overview study was conducted based on the Broome method. Broome's method is based on three steps in the search for texts, data evaluation and data analysis. So, in the search phase, post-retrospective study texts are examined in four stages in terms of inclusion criteria. After obtaining the conditions for entry into the study, the content of the study is evaluated and at the end of the data was analyzed.
\end{abstract}

Results: Ten articles were reviewed in this study that There was an association between diabetes and cancer and a serious risk for cancer. One of these studies has shown that diabetes is associated with an increased risk of breast cancer. Analysis of all 20 researches that studied in this study. Showed that women with diabetes (versus diabetic men) had a 20 percent increased risk of breast cancer.

Conclusion: There was a serious relationship between diabetes and various types of cancer in this study. Therefore, patients with diabetes need to have a special diet and take the necessary precautions. It should also be emphasized on the screening of breast cancer and other types of cancer by conducting tests and diagnostic work for the rapid diagnosis of cancer among diabetic patients. That to be provided the possibility of early detection of cancer and reduce mortality.

Keywords: Obesity-related cancer, Diabetes, cancer patients, diabetic patients

\section{Introduction}

Diabetes is a chronic metabolic disease that accounts for 15 percent of the health care costs in the United States. The prevalence of diabetes in
Iran is about $3-17 \%$, depending on the geographical area and 4 million Iranian people are at risk of it. Increasing your blood glucose can cause early and late complications of diabetes (17).

Address For Correspondence:

Mostafa Madmoli, Emergency Medical Technician, Dezful University of Medical Sciences, Dezful, Iran. E-mail: mostafamadmoli10@yahoo.com 
Complications of diabetes are very common among patients. Foot complications in people with diabetes have various muscular and neuropathic musculoskeletal disorders and circulatory disorders (8-14).Among the many complications of diabetes, it can be said that type 1 diabetes also affects the functioning and functioning of the digestive system. And the most common digestive symptoms include delayed stomach imagination, Gastroparesis, changes in the mobility of the small intestine, Gastroparesis with symptoms of anorexia, nausea, vomiting, premature somnolence and abdominal bloating. Lifestyle behaviors, including nutritional behaviors, play an important role in preventing and managing diabetes (15-21).

One of the disorders found in diabetic patients is depression. Which is one of the most debilitating problems in youth and adolescence $(22,23)$. Job stress is a combination of stressors and jobrelated situations which most people agree on being stressful. High occupational stress is known as a known psychosocial factor in cardiovascular disease $(24,25)$.

Diabetes also affects thalassemic patients. Thalassemia is a hereditary anemia. Blood transfusion has complications, some of which are related to immunologic complications and some Non-immunologic complications. (26-30).

In general, diabetes can cause various diseases, as well as a variety of cancers. Patients with diabetes have been reported to increase the risk of cancer by 20 to 25 percent compared to those without diabetes (31).

In this study, we intend to examine the relationship between these two diseases by examining several studies. Therefore, this systematic review was conducted to determine the relationship between diabetes and cancer and the risk of developing cancer in diabetic patients.

\section{Materials and Methods}

This study is a systematic review in order to achieve the goal of the study and to enhance the accuracy of its study and comprehension, this integrated overview study was conducted based on the Broome method. Broome's method is based on three steps in the search for texts, data evaluation and data analysis. So, in the search phase, post-retrospective study texts are examined in four stages in terms of inclusion criteria. After obtaining the conditions for entry into the study, the content of the study is evaluated and at the end of the data was analyzed.

The study, based on articles published in the last 25 years, was about the risk of developing cancer in diabetic patients. It was conducted by searching articles in search engines, SID, Magiran, Google Scholar, Embase, Science Fiction, Researchgate, Sciencedirect, PubMed in Persian and English. In the first stage, 38 articles were found. Of these, 14 articles related to the subject, published in the last 25 years, were reviewed.

Studies in line with the research goal They were written in English or Persian, access to their full text was possible and published over the last 25 years and unnamed studies were deleted without history and non-academic.

To achieve relevant studies, a wide range of keywords including Obesity-related cancer, Diabetes, cancer patients and diabetic patients was used as a one-to-one search, combined with the method "And" and "OR".

\section{Results}

Diabetes is a chronic metabolic disease that accounts for 15 percent of the health care costs in the United States. The prevalence of diabetes in Iran is about $3-17 \%$, depending on the geographical area and 4 million Iranian people are at risk of it. Increasing your blood glucose can cause early and late complications of diabetes (17). Patients with diabetes have been reported to increase the risk of cancer by 20 to 25 percent compared to those without diabetes (31). In this study, we want that by reviewing 14 articles, understand the link between diabetes and cancer.

Studies have shown that diabetes increases the chance of different types of cancer. For example, several studies have shown that diabetes increases the risk of colorectal cancer. In a study (32), the incidence of colorectal cancer in diabetic 
patients was 2.1 times higher than non-diabetic controls. And in another study, the odds of having colorectal cancer in diabetic patients were 1.23 times higher (33). Another study also found that diabetes increased the chances of developing colorectal cancer. In this study, 207 patients with colorectal cancer had 48 patients with diabetes in contrast, of the 207 controls, only 21 were diabetic. So in this study, diabetes can increase the chances of developing colorectal cancer (34).

In a study that was done, found that diabetes is associated with an increased risk of breast cancer. Analysis of all 20 studies studied in this study. Showed that women with diabetes (versus diabetic men) had a 20 percent increased risk of breast cancer. (35).

In a study, this study showed a significant relationship between type 2 diabetes and colon adenomas. Exposure to insulin and thiazolidinedione was associated with the formation of adenoma and none of the types of analgesic drugs and $\mathrm{HbA} 1 \mathrm{c}$ levels were predictive of adenoma (31).

In another study, more than 6,000 new cases were diagnosed. In men, there is a risk of total cancer in those with a history of diabetes Increased by $27 \%$. We also saw an increased risk of colon cancer and gastric cancer with borderline significance. In women, the incidence of cancer has increased significantly at the borderline, while the incidence of gastric cancer and liver cancer was statistically significant and the incidence of ovarian cancer was observed at the border (36).

The association between diabetes and primary liver cancer in a study, 59 cases with gallbladder cancer and bile ducts and 1502 controls in the hospital for non-acute illnesses Sixty-four cases of hepatocellular carcinoma have been reported in 87 diabetic patients (37).

In the study, there was no significant relationship between diabetes and gallstone, esophageal and stomach cancers. Diabetes was associated with an increased risk of developing liver, pancreatic, colon, and rectal cancers. The severity of diabetes, due to the length of time or the need for drug therapy, seemed to have a stronger association with the risk of developing liver, pancreatic and rectal cancers, but it did not have colon cancer (38).

In a study that was done, the risk of developing pancreatic and liver cancers among diabetic patients has increased. The increased risk of pancreatic cancer was fully apparent to people with diabetes. On the contrary, an increased risk of liver cancer was independent of the distance between diabetes and the diagnosis of cancer. There was no relation to esophagus, stomach, colon, rectum, lung, prostate, bladder, and kidney cancer. As a result, diabetes was associated with an increased risk of liver cancer among men (39).

In one study, diabetes mellitus was associated with an increased risk of colorectal neoplasm. The risk of colorectal cancer also increased significantly (40).

Of course, there was no significant relationship between diabetes and cancer in studies. For example, in a study by Dash et al. On African Americans, there was no significant relationship between diabetes and colorectal cancer (41).

According to studies such as (34), (42-44), A significant and positive relationship was found between high-fat diet and consumption of red meat with colorectal cancer.

\section{Discussion}

In general, diabetes can cause various diseases, as well as a variety of cancers. Patients with diabetes have been reported to increase the risk of cancer by 20 to 25 percent compared to those without diabetes. (31). In this study, we intend to examine the relationship between these two diseases by examining several studies. Therefore, this systematic review was conducted to determine the relationship between diabetes and cancer and the risk of developing cancer in diabetic patients.

According to the study (32-34), which showed diabetes mellitus increases the chances of developing colorectal cancer, colorectal cancer is a common and fatal illness with a high incidence of occurrence in different parts of the world and is the third leading cause of cancer deaths in the world (45). Cancer occurs when parts of the body 
begin to grow uncontrollably. One of the common cancers in women and men is colorectal cancer. Colorectal cancer is the fifth cancer in males and the third cancer in women in Iran $(46,47)$. The best way to prevent this disease is by feeding properly and using fiber in the diet. Constipation should be prevented if constipation is one of the most important causes of colorectal cancer.

Also, according to study (35), which showed diabetes was associated with an increased risk of breast cancer, and people with breast cancer at the age of menopause are more likely to have diabetes. Even statistics show that people with diabetes also have a chance of developing breast cancer, and about $20 \%$ of these people develop breast cancer. Lifestyle changes can reduce the risk of breast cancer. One of the factors that people with breast cancer are at risk for diabetes are estrogen resistance in the stages of chemotherapy. Using some medications such as glucocorticoid in chemotherapy can increase your blood glucose, use of these drugs is to prevent inflammation and nausea. When the body resists insulin, it is prone to spreading diabetes and a variety of cancers this may happen in people who have breast cancer. Also, in diabetics, with increased levels of insulin, breast tissue changes that increase the risk of developing breast cancer. Statistics show that people with advanced breast cancer and at the same time have diabetes, they have larger tumors than other breast cancer patients and in these people, the possibility of spreading the disease is also higher in the case of diabetes.

Also, in study (36), we saw an increased risk of colon cancer and gastric cancer with borderline significance. Overall, it can be said that the study showed that diabetes is associated with a serious risk of developing a variety of cancers.

\section{Conclusion}

There was a serious relationship between diabetes and various types of cancer in this study. Therefore, patients with diabetes need to have a special diet and take the necessary precautions. It should also be emphasized on the screening of breast cancer and other types of cancer by conducting tests and diagnostic work for the rapid diagnosis of cancer among diabetic patients. That to be provided the possibility of early detection of cancer and reduce mortality.

\section{Acknowledgment}

The author of this article is grateful to all those who have collaborated with me in this article.

\section{References:}

1. Moslemirad M, Madmoli M, Madmoli Y, Niksefat M. Prevalence of type 1 and type 2 diabetes and its related factors in diabetic patients hospitalized in Khatamol-Anbia hospital in Shoushtar, 2014-15: A retrospective study. Journal of Research in Medical and Dental Science. 2018 May 22;6(3):421-6.

2. Madmoli M, Eilami O, Rezaie K, Aliabad MA, Moslemirad M. Diabetes and the risk of suffering cardiovascular Diseases: A two-year retrospective study. International Journal of Ecosystems and Ecology Science (IJEES). 2018 Jun;8(3): 649-56.

3. Madmoli M Rostami F, Mirsami Yazdi N, Mosavi A, Baraz Sh. Evaluation of Prevalence of Diabetic Foot Ulcer and Its Related Factors in Diabetic Patients Admitted to KHatam-ol-Anbia Hospital in Shoushtar During 2015-2016: A Retrospective Study. International Journal of Ecosystems and Ecology Science (IJEES). 2018 June; 8 (3): 545-52.

4. Rostami F, Madmoli M, Mirsami Yazdi N, Baraz Sh. Evaluation of The Prevalence of Lower Limb Amputation and Its Related Factors in Diabetic Patients Admitted to KHatam-ol-Anbia Hospital in Shoushtar During The 2015-2016: A Retrospective Study. International Journal of Ecosystems and Ecology Science (IJEES). 2018 June; 8 (3): 553-60.

5. Raisifar Z, Afshar Nia A, Madmoli M, Madmoli Y. The Relationship Between Using Insulin and Suffering Alzheimer's Disease in Patients with Diabetes: A Two- 
Year Study. International Journal of Ecosystems and Ecology Science (IJEES). 2018 June; 8 (3): 623-28.

6. Shirali M, Madmoli Y, Roohafza J, Karimi $\mathrm{H}$, Baboli Bahmaei A, Ertebati S. Improvement diagnosis of diabetes using a combination of sugeno fuzzy inference systems and firefly algorithms. ijdld. 2017; 15 (3): 172-176. [in persian].

7. Raisifar Z, Afshar Nia A, Maghamesi Moarrefi H, Madmoli M. Evaluation of Gi Bleeding Prevalence and Its Related Factors in Diabetic Patients Hospitalized in KHatam-ol-Anbia Hospital During 2015-16: A Retrospective Study. International Journal of Ecosystems and Ecology Science (IJEES). 2018 June; 8 (3): 609-14.

8. Mostafa Madmoli, Zahra Kord, Azita Bandani, Negin Sedighi, Mahla Rezaei Shandiz, Pouriya Darabiyan, Alieh AfsharNia. Epidemiological and clinical study of patients with Alzheimer's in Five Cities of Khuzestan Province in 20162018. Medical Science, 2019; 23(95), 1-5

9. Hadi Mashali, Fatemeh Toleideh, Rezvan Rahmani, Pouriya Darabiyan, Mostafa Madmoli. The predictive role of Hyperlipidemia in the incidence of ACS in patients referring to Shahidzadeh Hospital in Behbahan in 2016 -2017. Medical Science, 2018; 22(94), 566-570

10. Mostafa Madmoli, Yasaman Modheji, Alireza Rafi, Rezvan Feyzi, Pouriya Darabiyan, Alieh AfsharNia. Diabetes and its predictive role in the incidence of Alzheimer's disease. Medical Science, 2019; 23(95), 30-34

11. Madmoli M. Quality of Life in Patients with Cancer and Some Factors Affecting it: A Systematic Review. International Journal of Research Studies in Science, Engineering and Technology. 6(1), 2019; 1-7

12. Madmoli M. Clinical and Laboratory Findingin Children with Leukemia: a Systematic Review. International Journal of Research Studies in Science,
Engineering and Technology, vol. 5, no. 10, pp. 1-6, 2018.

13. Madmoli M, Madmoli $Y$, Khodadadi M, Samsamipour M. Some Factors Affecting Quality of Life in Patients with Diabetes: A systematic Review. Annals of Microbiology and Infectious Diseases. 2019; 2(1). 26-30.

14. Mostafa Madmoli, Mehdi Fallah bagher shaidaei, Akram Rohani, Pouriya Darabiyan, Fariba Mobarez. The correlation between alcohol consumption and reducing the age of cancer incidence in patients with this disease. Medical Science, 2019, 23(95), 48-53

15. Mostafa Madmoli, Zahra Mahmoudi Dehcheshmeh, Alireza Rafi, Zahra Kord, Fariba Mobarez, Pouriya Darabiyan. The rate of some complications and risk factors of diabetes in diabetic patients: Study on cases of 3218 diabetic patients. Medical Science, 2019; 23(95), 63-68

16. Mostafa Madmoli. Evaluation of Chemotherapy Complications in Patients with Cancer: A systematic Review. International Journal of Research Studies in Science, Engineering and Technology, vol. 5, no. 12, pp. 59-64, 2018.

17. Mostafa Madmoli, Mehran Yarbig, Negin Sedighi, Pouriya Darabiyan, Fariba Mobarez. Communication between body mass index and the risk of obesity-related cancer: A 5-year study on patients with cancer. Medical Science, 2019; 23(95), 6974.

18. Madmoli M. Quality of Life in Patients with Cancer and Some Factors Affecting it: A Systematic Review. International Journal of Research Studies in Science, Engineering and Technology. 6(1), 2019; 1-7

19. Madmoli M, Madmoli Y, Khodadadi M, Samsamipour M. Study of Some Effective Treatments for Accelerating Diabetic Foot Ulcer Healing: A Systematic Review. International Journal of Research Studies in Science, Engineering and Technology, 6(2), 2019; 34-39 
20. Madmoli M, Madmoli Y, Khodadadi M, Samsamipour M. Factors Affecting the Level of Glycosylated Hemoglobin in Patients with Diabetes: A Systematic Review. Annals of Microbiology and Infectious Diseases 2(1), 2019; 43-47.

21. Madmoli M, Madmoli Y, Khodadadi M, Samsamipour M. Some Factors Affecting Quality of Life in Patients with Diabetes: A systematic Review", Annals of Microbiology and Infectious Diseases, 2(1), 2019; 26-30.

22. Madmoli Y, Madmoli M, Qashqaei nezhad N, Bosak S. Prevalence of depression and associated factors in adolescents of masjedsoleyman. JPEN. 2016; 2(4):31-40.

23. Madmoli M, Madmoli Y, Bigdeli Shamloo MB, Etebari A, Mahmoodi Kouhi A, Azami M. The Relationship Between Depression and Religiousness in Female High School Students of Masjed Soleyman in 2015. Journal of Pediatric Nursing. 2017 Jun 15;3(4):15-22.

24. Roughani A, Madmoli M, Raisifar Z, Kikhavani S, Yasemi M, Azami M, Sharifi Z. The prevalence of behavioral disorders and its related factors in elementary school children in Ilam City in 20112012. Indian Journal of Forensic Medicine \& Toxicology. 2018;12(4):165-9.

25. Madmoli M, Nikpay S. An Investigation of the Relationship between Spiritual Health and Depression, Anxiety, and Stress among Students of ilam University of Medical Sciences. Journal of Research in Medical and Dental Science. 2018 May 17;6(3):294-300.

26. Madmoli Y, Aslani A, Ahmadi Y, Mousavi M, Mashalchi H, Niksefat M, Madmoli M. Study Habits and Related Factors in Students of Nursing and Midwifery College of Dezful University of Medical Sciences in 2015. Iranian Journal of Nursing Research (IJNR). 2017.

27. Madmoli Y, Akhaghi Dezfuli SM, Beiranvand R, Saberi Pour B, Azami M, Madmoli M. An epidemiological and clinical survey of patients with $\beta$ thalassemia in dezful in 2015. Iranian
Journal of Epidemiology. 2017;13(2):14552.

28. Maraghi E, Adavi A, Madmoli Y, HeidariSoureshjani R, Madmoli M. The Effect of Orem Self-Care on Mental Health of Patients with Thalassemia Major. Journal of Clinical Nursing and Midwifery. 2018;4(3).

29. Madmoli M, Madmoli Y, Rahmati P, Adavi A, Yousefi N, Gheisari Z, Abbaszade Aliabad M. Quality of Life and Some Related Factors in Patients with Beta Thalassemia Major in Southwest Iran. Journal of Client-centered Nursing Care (JCCNC. 2017;3(2).

30. Madmoli Y, Akhaghi Dezfuli SM, Adavi A, Maraaghi E, Heidari Soureshjani R, Madmoli M. The Effect of Orem Self-Care on Mental Health of Patients with Thalassemia Major. Journal of Clinical Nursing and Midwifery. 2018 Jul 1;7(2):108-15.

31. Vigneri P, Francesco F, Laura S, Giusepp P, Richado V. Diabetes and cancer. Endocr Relat Cancer 2009; 16:1103-23.

32. Wang JY, Chao TT, Lai CC, Wang CY, Wu VC, Wang SM, Lin HI, Tang SH, Lin HW. Risk of colorectal cancer in type 2 diabetic patients: a population-based cohort study. Japanese journal of clinical oncology. 2013 Jan 3;43(3):258-63.

33. Bosetti C, Rosato V, Polesel J, Levi F, Talamini R, Montella M, Negri E, Tavani A, Zucchetto A, Franceschi S, Corrao G. Diabetes mellitus and cancer risk in a network of case-control studies. Nutrition and cancer. 2012 Jul 1;64(5):643-51.

34. Azizi H, Delpisheh A, Sayehmiri K, Asadollahi K, Esmaeili ED, Sharbafi J. Risk of Colorectal Cancer in Type2 Diabetic Patients. Majallah-i pizishki-i Danishgah-i Ulum-i Pizishki va Khadamat-i Bihdashti-i Darmani-i Tabriz. 2016 Oct 1;38(4):56.

35. Eddi R, Karki A, Shah A, DeBari VA, DePasquale JR. Association of type 2 diabetes and colon adenomas. Journal of gastrointestinal cancer. 2012 Mar 1;43(1):87-92. 
36. Inoue $M$, Iwasaki M, Otani T, Sasazuki S, Noda M, Tsugane S. Diabetes mellitus and the risk of cancer: results from a largescale population-based cohort study in Japan. Archives of internal medicine. 2006 Sep 25;166(17):1871-7.

37. La Vecchia C, Negri E, Decarli A, Franceschi S. Diabetes mellitus and the risk of primary liver cancer. International journal of cancer. 1997 Oct 9;73(2):204-7.

38. Luo J, Chlebowski R, Liu S, McGlynn KA, Parekh N, White DL, Margolis KL. Diabetes mellitus as a risk factor for gastrointestinal cancers among postmenopausal women. Cancer Causes \& Control. 2013 Mar 1;24(3):577-85.

39. Rousseau MC, Parent MÉ, Pollak MN, Siemiatycki J. Diabetes mellitus and cancer risk in a population-based casecontrolstudy among men from Montreal, Canada. International journal of cancer. 2006 Apr 15;118(8):2105-9.

40. Luo S, Li JY, Zhao LN, Yu T, Zhong W, Xia ZS, Shan TD, Ouyang H, Yang HS, Chen QK. Diabetes mellitus increases the risk of colorectal neoplasia: an updated metaanalysis. Clinics and research in hepatology and gastroenterology. 2016 Feb 1;40(1):110-23.

41. Azizi H, Asadollahi KH, Davtalab Esmaeili E, Mirzapoor M. Iranian Dietary Patterns and Risk of Colorectal Cancer. Health Promot Perspect 2015; 5(1): 72-80.

42. Ulaganathan V, Kandiah M, ZalilahMS. Colorectal Cancer and its Association with the Metabolic Syndrome: a Malaysian Multi-Centric Case-Control Study. Asian Pacific Journal of Cancer Prevention 2012; 13:73-77.

43. Hosseinzadeh A, Daraei A. Environmental Factors Associated with Sporadic Colorectal Cancer. Esfahan, Iran J Health System Research 2012; 8(2):229-236.

44. Safari A, MohdShariff Z, Kandiah M. Dietary patterns and risk of colorectal cancer in Tehran Province: a case-control study. J BMC Public Health 2013; 13: 222.

45. Maajani K, Khodadost M, Fattahi A, Shahrestanaki E, Pirouzi A, Khalili F,
Fattahi H. Survival Rate of Colorectal Cancer in Iran: A Systematic Review and Meta-Analysis. Asian Pacific Journal of Cancer Prevention. 2019 Jan 1;20(1):1321.

46. Baghestani AR, Daneshvar T, Pourhoseingholi MA, Asadzade $\mathrm{H}$. Survival of colorectal cancer patients in the presence of competing-risk. Asian Pacific Journal of Cancer Prevention. 2014;15(15): 62-68

47. Pourhoseingholi MA, Zali MR. Colorectal cancer screening: Time for action in Iran. World J Gastrointest Oncol. 2012;4(4):823.

How to cite this Article: Mostafa Madmoli., A Systematic Review Study on the Communication between Diabetes and Cancer: The Serious Risk of Cancer in Diabetic Patients. Int. Res. Med. Health Sci., 2019; 2(2):1-7.

Source of Support: Nil, Conflict of Interest: None declared. 